\title{
An orchestration approach to smart city data ecosystems
}

\author{
Anushri Gupta \\ Queen Mary University of \\ London
}

\author{
Panos \\ Panagiotopoulos
Queen Mary University
of London \\ Panagiotopoulos
Queen Mary University
of London \\ Panagiotopoulos
Queen Mary University
of London
}

Frances Bowen

University of East

Anglia

\begin{abstract}
Research on smart cities has illustrated the use of data analytics, open data, smart sensors and other data-intensive applications that have significant potential to transform urban environments. As the complexity and intensity of these projects has increased, there is a need to understand smart city data ecosystems as an integrated view of data applications by the various city entities that operate within an institutional environment. This paper examines how authorities involved in such ecosystems coordinate data initiatives from an orchestration perspective. A case study of London's city data initiatives highlights the challenges faced in complex city data environments and the importance of an integrated view. Three elements of orchestration in smart city data ecosystems - namely openness, diffusion and shared visionare identified as the main enablers of city data initiatives within London's local government authorities. The study contributes to our theoretical understanding of orchestration within data ecosystems, as well as the social and technological impacts of city data.
\end{abstract}

Keywords: smart cities; data ecosystems; orchestration; local government; London city data

\section{Introduction}

Research on smart cities has attracted considerable attention due to the large interest in the socio-technological aspects of urban development (Appio et al., 2019; Lee et al., 2014; Moutinho and Heitor, 2007). Several studies have contributed to our knowledge of how new data applications can generate promising benefits in the urban environment, for example, Internet of Things (Bresciani et al., 2018), social media analytics (Batty et al., 2012), open innovation and crowdsourcing (Cohen et al., 2016) or open government data (Jetzek et al., 2014). As the variety and complexity of these applications has increased, there is a need for studies to approach the domain from a holistic viewpoint, taking into consideration the institutional and contextual conditions in which cities operate (Blazquez and Domenech, 2018; Lee et al., 2014; Meijer, 2018; Visnjic et al., 2016). The relative lack of attention to such integrated views of city data projects and their management aspects have resulted in rather isolated views on how these initiatives can support local communities and improve urban environments (George et al., 2014; Hashem et al., 2016; Mora et al., 2019; Nilssen, 2019). 
The missing narrative addresses how data applications and systems can be scaled across wider city entities to enable consistency in value creation from different projects. The approach of city data has become even more relevant in metropolitan areas with devolved powers or federalised structures. As a strategic resource, "city data is that which is held by any organisation...... bearing on local populations and the functioning of that place. It can be static, near-real time or in the future, real time, descriptive or operational" (EIP-SCC and BSI, 2016, p. 6). City data at its core is an approach to reduce friction to data sharing across city entities, and to rebalance the 'government-technology' industry narrative around local government data (British Standards Institution, 2015).

A city data view consolidates integrated environments with their respective management and policy challenges. Among varied entities, local and regional government authorities are usually identified as the largest steward of city data with data stored across various legacy systems both in-house, and in systems commissioned by external vendors (Malomo and Sena, 2017; British Standards Institution, 2015). Further to these public actors, a network of industry and government-backed SMEs, service delivery providers, think-tanks and other intermediary organisations are usually critically involved with city data projects (Cohen et al., 2016; Hong et al., 2019; Malomo and Sena, 2017; Snow et al., 2016). Altogether, these organisations, the city data and their interactions can be conceptualised as a smart city data ecosystem.

In this article, we develop an orchestration perspective to conceptualise the complex management challenges within smart city data ecosystems. While there is a good understanding of orchestration within business networks, wherein the dominant hub entity co-ordinates efforts and resources of network members towards a common goal (Dhanaraj and Parkhe, 2006; Nambisan and Sawhney, 2011), less is known about how orchestration can apply within local governance ecosystems or complex data projects. Studies note that local governments need to be more focused and there is a need for city actors to be able to co-ordinate as well as collaborate to mobilise the data ecosystem towards desired ends (Dawes et al., 2016; Suzuki and Finkelstein, 2019). Despite these noteworthy statements, an empirical investigation into the same in currently missing. We thus adopt an orchestration perspective to illustrate the mechanisms through which entities in smart city data environments coordinate data-related initiatives and maintain a shared and unified view of city data, their applications and intended value.

To empirically examine how orchestration occurs within smart city data ecosystems, we present a case study of London's city data. The study draws its findings on primary and secondary sources of data from a variety of perspectives that bring together an understanding of London's complex institutional and technological environment within the period 2017-2019.

Orchestration mechanisms within the case study were manifested through three different elements - openness, diffusion and shared vision - which shaped the environment to foster city data initiatives. Although orchestration took place within London's institutional structures, the analysis showed the simultaneous presence of both individual and collective orchestrators with mutual interests in data leadership and implementation. This analysis provides a different perspective to the more traditional view of orchestration on the basis of authority, power or control of resources (Dhanaraj and Parkhe, 2006; Giudici et al., 2018; Hong and Snell, 2013). It further contributes to studies of government or public sector orchestration in managing complex projects by highlighting the duality of an orchestrator as both a strategist and manager, 
in contrast to covening and facilitation role (Janssen and Estevez, 2013; Shaw et al., 2019). From a policy perspective, this is one of the first studies that highlight the impacts of data on the dynamics of interaction between city entities. It particularly explores how smart city data projects can be more holistically integrated in local governance beyond the excitement and success of pilot projects.

The remainder of the paper first discusses city data as a core element of smart cities in Section 2. This is followed by Section 3 that lays the background on orchestration and its importance as a theoretical lens within smart city data ecosystems. The case study methodology and relevant sources of data are discussed in Section 4, followed by the findings and analysis in Section 5. The discussion in Section 6 develops theoretical and policy contributions including how multi-layer tensions in smart city data ecosystems are orchestrated and highlights the dynamics of interaction between city entities. Conclusions are briefly outlined in section 7 .

\section{Smart cities and city data ecosystems}

Increasing pressures on city resources along with growth in urbanisation have resulted in significant interest in data-intensive smart city applications (George et al., 2014; Van Zoonen, 2016). As a result, local governments and metropolitan authorities worldwide have been deemed responsible for designing and implementing smart city strategies and initiatives. The strategic priority of these initiatives has been enabled by the establishment of consortia of smart city standards and assessment frameworks ( see for example - Smart Cities | BSI Group, no date; EIP-SCC, no date; World Council on City Data, no date).

Smart cities are an established field in the academic scholarly literature, with studies putting forth various interdisciplinary perspectives from digital city (Ishida and Isbister, 2000) to intelligent city (Komninos, 2011), ubiquitous city (Shin, 2009) and smart city (Appio et al., 2019; Nam and Pardo, 2011) to name a few. Although there is no consensus around the definition of a smart city, several studies have outlined the key dimensions (Chourabi et al., 2012; Giffinger et al., 2007; Jucevipius et al., 2014; Nilssen, 2019). From a data perspective, the majority of studies have identified experimental and pilot smart use cases like Internet of Things applications (Bresciani et al., 2018), social media data analytics (Batty et al., 2012), open innovation and crowdsourcing (Cohen et al., 2016) and open government data (Jetzek et al., 2014; Pereira et al., 2017). Data from these large systems and the underlying transactions that take place in cities everyday have resulted in a data 'deluge' that is further intensified with data held in legacy systems by local and regional governments (British Standards Institution, 2017; Vogl et al., 2019).

As data applications in smart cities are moving towards consolidation, researchers have begun to investigate the deployment of urban data platforms to manage and coordinate the provision of city data (Berrone et al., 2016; Danneels et al., 2017; Suzuki and Finkelstein, 2019). Such platforms broadly include closed and open data whose value creation potential might defer. Closed data platforms remain within the sole control of city authorities so that data can increase organisational value, for example, by using surveillance using generated from CCTV cameras (Van Zoonen, 2016). In contrast, open platforms allow for public access to, 
modify and share data on the platform and are for "more collaborative models of smart city governance that emphasise a role for city governments in the curation and management of data assets to support a city's strategic priorities" (Barns, 2018, p. 1). Open data platforms are often supported by enabling tools like 'city dashboards', 'datastores' and 'data marketplaces' (Barns, 2018). Irrespective of the approach, known data challenges for urban platforms arise with vendor-lock in problems, misalignment of vendor-government needs, interoperability challenges, complicated data transformation process and fragmented data supply chains (Moshrefzadeh et al., 2017; Suzuki and Finkelstein, 2019)

In light of the above challenges, the narrative of city data has moved from platforms to more consolidated infrastructures that provide a wide variety of data to city actors according to their needs using distributed and decentralised architectures (Estermann et al., 2018; Klievink et al., 2017; Moshrefzadeh et al., 2017). This conceptualisation is not only limited to the technology powering these platforms (hard infrastructure) (Blazquez and Domenech, 2018); but also includes their value networks and governance aspects (soft infrastructure) (Jetzek, 2016; Suzuki and Finkelstein, 2019). When matching data architectures with institutional rules, certain new roles emerge that city entities have to fulfil (e.g. data collection, data storage, data usage, data visualisation, data access) - this interplay is conceptualised by Meijer (2018) as 'datapolis'. Meijer (2018) highlights the transformative impact of city data as 'data-infrastructures embody value judgments about the city and thus are not neutral tools but mechanisms for governing the political community... a form of meta-governance" (Meijer, 2018, p. 196).'

Therefore, smart city data ecosystems embody new interactions between all entities involved with data from local authorities to developers, technology providers, citizens and other users. This conceptualisation of city data ecosystems underlines an integrated view of data applications within their institutional environment. However, questions remain on how smart city data ecosystems are organised or orchestrated, which we address in the next section.

\section{Theoretical perspective: Orchestration of city data}

City data ecosystems are multi-layered and exist within the operating institutional environment of varied hierarchies and cultures. This raises challenges identified in the literature such as how dominant city entities legitimise their activities (Chatfield and Reddick, 2017) or develop new entities to overview initiatives like the New York Mayor's Office of Data Analytics (Copeland, 2015). A credible theoretical lens to conceptualise these integrated and complex environments requires a deep understanding of the coordination mechanisms that will create a credible environment for the sustained implementation of city data projects.

Originating from the management literature, orchestration has been a fundamental concept to understand the evolution of networks and ecosystems. Network orchestration is the process of coordinating initiatives of a network of organisations towards a collective goal (Dhanaraj and Parkhe, 2006; Nambisan and Sawhney, 2011). Network orchestration has been well examined within business ecosystems or innovation networks with a focus on the optimal use of shared resources and resource complementarities (Clegg et al., 2016; Dagnino et al., 2016; Paquin and Howard-Grenville, 2013). Key orchestration activities identified to achieve 
these objectives are value appropriation, legitimising and knowledge mobility (Dhanaraj and Parkhe, 2006; Nambisan and Sawhney, 2011; Perks et al., 2017). These activities are orchestrated by a primary actor that is identified as an organisation with dominance and authority in the network, usually referred to as a 'hub' firm (Dhanaraj and Parkhe, 2006; Ford, 2011; Huxham and Vangen, 2000; Pikkarainen et al., 2017). Researchers have applied network orchestration as a theoretical lens in different types of networks. Giudici et al. (2018) introduce the concept of open system orchestration in entrepreneurial networks wherein the aim of the orchestrator is supporting network members in their varied objectives that altogether facilitate "spontaneous knowledge sharing and discovery of complementarities" (Giudici et al., 2018, p. 1395). In contrast, closed network orchestration is about directly implementing the motivations of the dominant hub firm (Parmentier and Mangematin, 2014).

In digital government projects, the concept has been extended to government orchestration around a platform ecosystem (Cordella and Paletti, 2019). Platforms are the means to orchestrate public-private stakeholder relationships managed by government entities (Shaw et al., 2019), with the overarching aim of being efficient and achieving 'more with less' (Janssen and Estevez, 2013). The government orchestration perspective assumes the central role of one government authority that coordinates the development of resources and capabilities within the network. One of the potential benefits of government organisations as orchestrators is that the platform approach enables to decouple 'row' functions like service delivery from 'steer' functions like policy making (Shaw et al., 2019).

Overall, the role of the orchestrator in business, entrepreneurial and government networks has been perceived as a single dominant organisation that coordinates the network by linking actors through the activities and structural elements of the network. Orchestration in complex multi-actor ecosystems remains less explored although the practice of the concept in those environments can be fundamentally different (Jacobides et al., 2018). Dynamic environments enabled or constrained by institutional structures can vary a lot from interactions across organisational boundaries (Oparaocha, 2015; Tsujimoto et al., 2018) due to the varied actors in the ecosystem each with their own priorities, bureaucracies and cultures.

City data ecosystems represent a complex version of multi-actor ecosystems that challenge city actors. Visnjic et al. (2016, p. 137) refer to ecosystem orchestration in smart cities as "the dynamic ability to carefully select and implement the appropriate ecosystem structure for the provision of specific services or the development of complex projects, and to reconfigure and adjust this structure according to how city stakeholder needs evolve." They identify two approaches to orchestration in cities as 'extended enterprise' and 'platform markets'. The first forms around a business ecosystem that integrates inputs from service organisations, whereas the second provides a platform view of interactions between third parties and citizens as facilitated by city governments. Despite the significance of the above study, our current understanding of orchestration is limited to business and platform ecosystems that highlight resource exploitation, dominance and authority of a 'hub' firm, and the platform as a mediator of relationships. There certainly lies scope to unravel the potential of orchestration as a conceptual framework to inform management practice in dynamically interacting and institutionally influenced city data ecosystems.

Our study thus draws on the orchestration perspective to address the following question: "How can orchestration explain the coordination and implementation of smart city data 
ecosystems?" A new understanding of this question will illustrate the challenges of coordinating, developing and transferring data assets and skills across city wide entities. It will further address issues of leadership within city data ecosystems and how they relate to multilayer tensions. Insights from the study can allow city managers to sense the dynamics of the ecosystem and mechanisms through which orchestration can be effectively enabled. At the conceptual level, the study can extend the concept of orchestration within the public sector and, more specifically, within local governance.

\section{Research methodology}

The study adopts a qualitative case study approach to explore how an orchestration perspective can contribute to our understanding of an integrated view of city data environments. London's ecosystem of city data initiatives was chosen as a suitable research setting for several reasons (Yin, 2009). London represents a highly complex institutional environment that allows an indepth analysis of how actors orchestrate the ecosystem to create value from city data. The complexity and scale of London's city data initiatives presents a unique and revelatory case that is indicative of the processes taking place in many city environments. The timing of this study within 2017-2019 has been a crucial driver for the maturity of London's city data agenda and its new transformation phase that has involved all London's local authorities. By observing and interrogating these processes, the researchers had access to both primary and secondary data.

\subsection{Research setting}

London's complicated local government landscape is led by the Greater London Authority (GLA) as the main city administration body. The GLA provides metropolitan oversight to 33 individual local government authorities known as the London Boroughs (LBs) that function as the main citizen-facing bodies and are each responsible for over 600 services. The GLA is responsible for delivering the Mayor's strategic objectives like planning and promoting the economic and social development of the capital. It also acts as the parent organisation for several functional bodies including Transport for London (TfL), the city's transport provider, and the police, ambulance and fire services across the metropolitan area. The GLA does not directly deliver services and does not hold large amounts of transactional and relational data, which are usually with the functional bodies.

Other primary actors involved with smart city data across London include think-tanks, advocacy and research organisations including the central government-backed Future Cities Catapult (FCC), the innovation foundation Nesta, and the Open Data Institute (ODI). Furthermore, there is strong presence of the industry technology sector and the developer community including many established and start-up companies that specialise in urban data, data analytics and other smart city applications. Figure 1 outlines a timeline of key events, and Figure 2 provides an overview of the current state of London city data.

One of the key data assets in the London city data ecosystem is the London Datastore run by the GLA. Initially launched in 2010 and revamped in 2014, it was one of the first open government data portals in the world. With presently over 4,000 registered users, 
approximately 50,000 visitors every month and 700 datasets available, the London Datastore has been playing a pivotal role in shaping the city data debate and ecosystem in London.

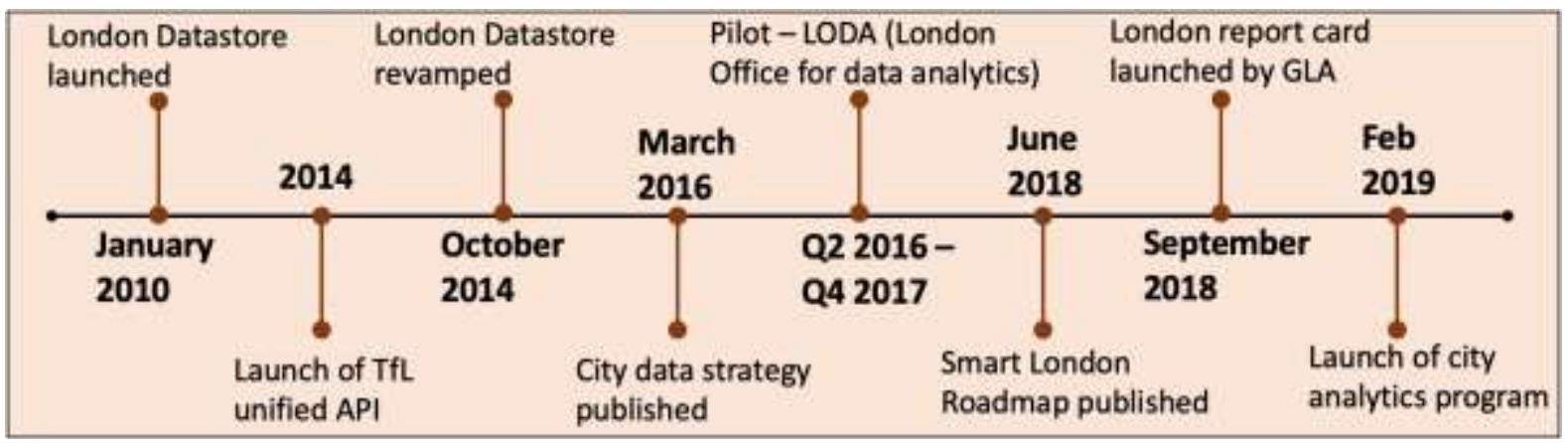

Figure 1: Timeline of milestones in London city data implementation

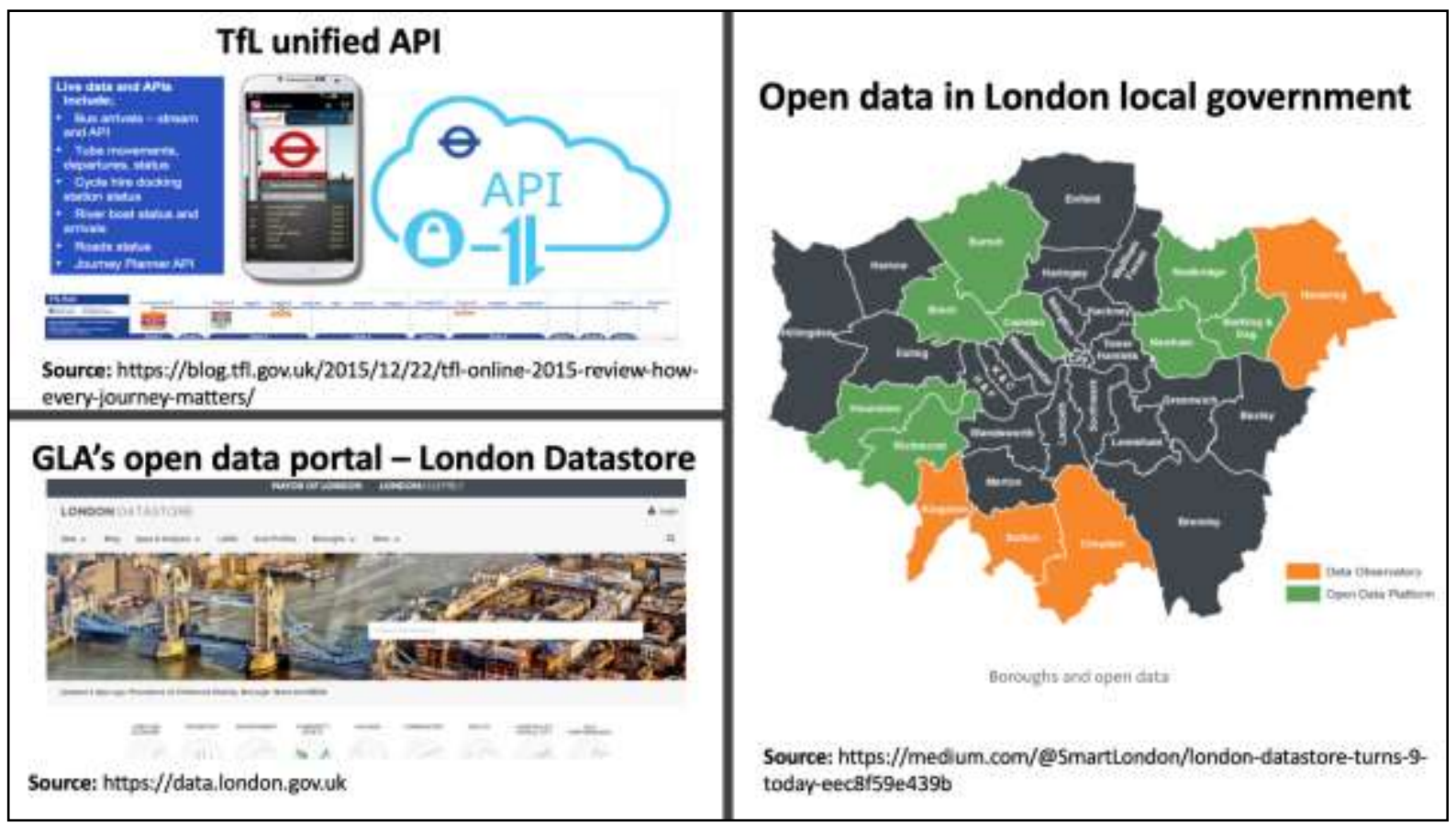

Figure 2: Current state of London city data

Data from the Datastore mainly consists of aggregated analytical data (summary statistics) that are compiled periodically to inform policy and strategy at the GLA. It also publishes London-specific datasets that appear in the national data portal data.gov.uk. Other London Boroughs have implemented their own versions of open data portals and platforms either individually or in joint partnerships (e.g. tri-borough partnerships). Furthermore, the functioning bodies - especially Transport for London ( $\mathrm{fLL}$ ) - made the distribution of their 
datasets available through a variety of formats and public APIs. This complexity can be attributed to the mere size and variety of data from all of London's authorities and providers.

While each data initiative creates its own organisational value and possibly value to the public, an integrated view of London's city data made sustained collaboration and maintenance a complicated challenge. To address this, the London City Data Strategy launched in 2016 (Greater London Authority, 2016) and the Smart London Roadmap set out in 2018 (Greater London Authority, 2018), identify city data as a core element to put in place structures and mechanisms to target collaboration deficits. The aims of these strategies are twofold: 1) to accelerate data provision in the ecosystem by shifting the focus from data release on numerous portals to data quality and data interoperability; 2) to maximise service delivery and data collaboration in the ecosystem whilst improving internal operations against the constraints of data quality. In essence, London city data is an approach to develop a sense of consistency across London's public services.

\subsection{Data collection and analysis}

Data collection for the study took place between April 2017 and May 2019. This period offered the opportunity to observe the consolidation of London's city data initiatives when the Greater London Authority (GLA) along with the other London authorities and the wider third sector, were scoping and prioritising city data initiatives for London.

The data sources comprised of interviews, participant observation and secondary data in the form of documents, videos, policy reports, datasets and other sources that supported triangulation (Yin, 2009). Primary data were collected from March 2018 to May 2019 with 23 semi-structured interviews conducted with key informants across the London's city data ecosystem as outlined in Table 1. Snowball sampling and personal invitations were used to select the interviewees responsible for shaping the London city data ecosystem (Scott and Glaser, 2006). The majority of interviews lasted between 60 and 90 minutes and were recorded and transcribed fully. The interview guide with general questions and questions more specific to each type of informant can be found in appendix A.

Table 1: Case study database (primary data)- interviews

\begin{tabular}{lll}
\hline Type of actor & Role & Interviews \\
\hline City administration & $\begin{array}{l}\text { Policy analysts; City data project lead and manager; } \\
\text { LODA board members; Smart London Board }\end{array}$ & 5 \\
$\begin{array}{l}\text { London Boroughs } \\
\text { (9 out of 33) }\end{array}$ & $\begin{array}{l}\text { Head of policy and insight; Data and insight } \\
\text { managers; Corporate information managers; } \\
\text { analyst; service director }\end{array}$ & 10 \\
$\begin{array}{l}\text { Transport for London } \\
\text { (TfL) }\end{array}$ & Open data manager in data and digital team & 1 \\
$\begin{array}{l}\text { Advocacy } \\
\text { organisations and } \\
\text { private tech sector }\end{array}$ & $\begin{array}{l}\text { Smart city standards lead; Project managers; } \\
\text { Director of government innovation team; head of } \\
\text { local public service; Founder and CEO of SME }\end{array}$ & 7 \\
\hline
\end{tabular}


Participant observation data were collected from November 2017 to March 2019 as detailed in Table 2. The timing of conducting this study has been crucial to collecting data by observing workshops, events, and conferences, and participating in unconference sessions ${ }^{1}$ run by the local government. The lead author attended these events and detailed notes of activities, informal discussions and decision-making processes were taken.

Secondary data were collected from April 2017 to May 2019. Besides enriching the case study database, archival data and blogposts informed the current ongoing practice and supported the design of opening questions of the interview guide catered to each interviewee's role. These sources included policy documents and reports (22), blogposts by key actors (50), presentation slides (15) and videos (9). These sources also assisted in obtaining granular verification of facts and enhancing validity of insights.

Table 2: Case study database (observation data) - events

\begin{tabular}{ll}
\hline Type of events & Number \\
\hline Public sector and industry conferences & 5 \\
Unconference & 3 \\
Events & \\
$\quad$ Roundtable discussions & 1 \\
$\quad$ Talks and Panel discussions (half day to 2-3-hour events) & 10 \\
Workshops & 1 \\
\hline
\end{tabular}

Data analysis occurred simultaneously with the data collection phase for the initial 12 interviews. This allowed to identify gaps in the data and modify the interview guide. The data were analysed between stages of collection and in their entirety after collection was completed. Overall, an inductive approach was used to construct the theoretical model in light of empirical evidence (Bhattacherjee, 2012; Miles \& Huberman, 1994). Accordingly, the analysis progressed through multiple iterative steps that are outlined below for simplicity.

The data were analysed using NVivo inductively for themes that were coded as first order concepts; wherein the themes reflected the language and concepts used by informants (Gephart, 2004). The search for first order concepts were focussed on activities, governance structures, operating mechanism and incentives that support the functioning of city data. Axial coding was implemented in the next phase of the analysis to progress towards a more theory-driven explanation (Miles \& Huberman, 1994).

\section{Findings}

Figure 3 shows the main themes of orchestration identified in the case analysis. The three overarching dimensions that operate at an ecosystem level are summarised as openness, diffusion and shared vision. 'Openness' allows ecosystem actors to sense and replicate city data capabilities present in the ecosystem, 'diffusion' is a mechanism to seed advanced data

\footnotetext{
${ }^{1}$ An unconference is an interactive session that allows practitioners and participants to engage and learn from each other. For example, the Smart London Camp held in April 2018 informed the development of the Smart London Roadmap, and was attended by the lead author.
} 
skills and build trust in the wider city data ecosystem through the implementation of knowledge diffusing liberating structures and agile delivery of projects at some parts of the ecosystem. Moreover, we identify 'shared vision' as a rather mature stage of orchestration that involves delivering consistency and best practice in the ecosystem through setting up of central coordination structures orchestrated by the regional authority GLA. The activities under this dimension are structured, planned and governed for a pan ecosystem level, in contrast to initiatives under 'diffusion' that are ad-hoc at an ecosystem level limited to activities of specific organisations that are either trialling innovative approaches or are advanced city data users in the ecosystem.

\section{$1^{\text {st }}$ order codes}

- Application Programming Interfaces (APIs) to meet user needs and support data interoperability

- Open standards to enable data sharing across ecosystem, and ensure access to data in procured IT systems

- Open source technologies to enable replication and validation of data initiatives $2^{\text {nd }}$ order codes

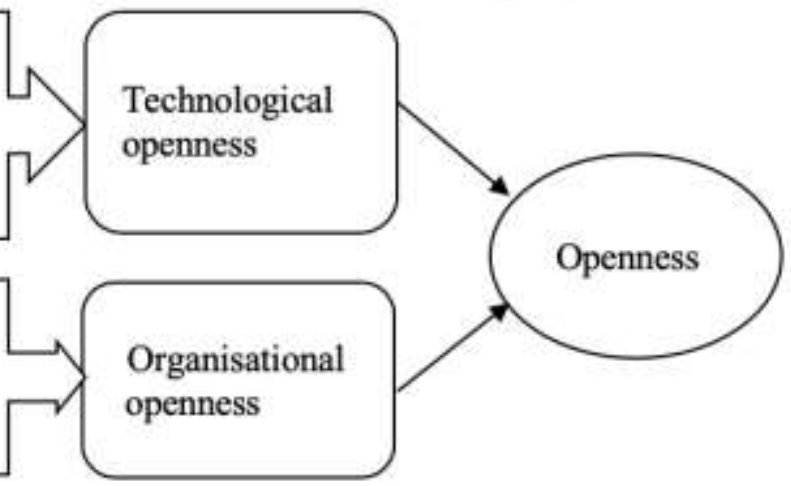

- Engagement \& transparency via open data portals

- Working in the "open't to share plans and experiences with wider community

- Demonstrate a clear \& open value exchange between government \& SME - e,g. hackathons

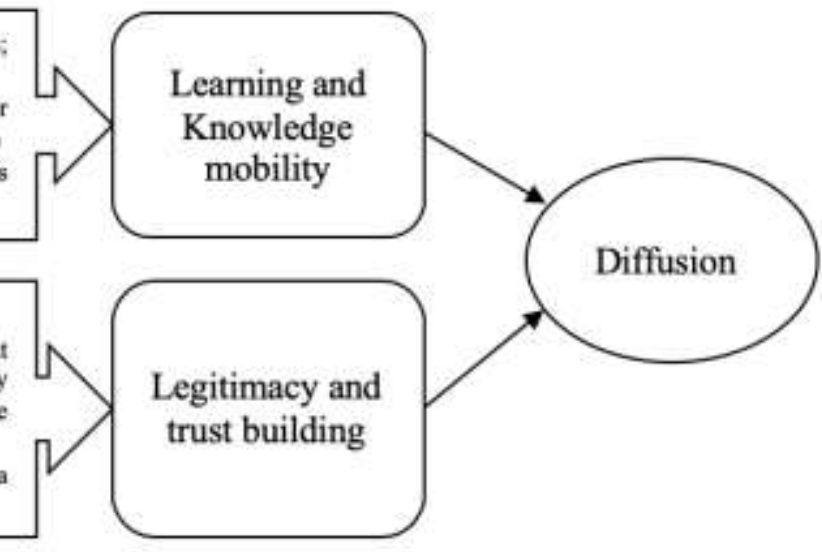
embedded work practices and co-location

- Ad-hoc structures with voluntary participation for knowledge exchange, example Borough data partnership

- Convening analytical network to ensure access to skills and expertise, as and when needed.

- Agile development of projects for 'quick wins'

- Problem-based procurement-a pro-active approach that helps government engage with many novel SMEs, by specifying the problem to be solved rather than stating the product needed.

- Implementing pilot projects to demonstrate potential of a new data initiative.

- Defining norms that represent the values of the wider ecosystem

- Boards with representation from various backgrounds to facilitate joint strategic decision making

- Crafting incentives to mobilise ccosystem actors towards a shared vision

- Define deliverables

- Developing institutions to provide support in core capabilities - e.g. City analytics program; Data academy

- Co-design of the London Planning development database

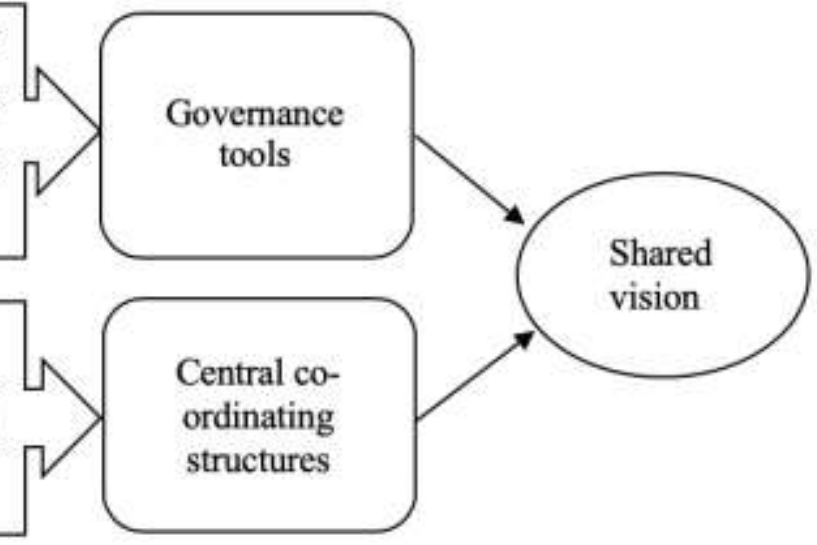


Figure 3: Overview of main themes and dimensions of city data orchestration

\subsection{Openness}

The analysis highlights the importance of being 'open' in the smart city data ecosystem as a driver to implement the city data initiatives efficiently. Conceptually, this includes managing technological and organisational openness that allows the orchestrator to demonstrate a sense of leadership and for ecosystem members to leverage successful initiatives and share insights.

\subsubsection{Managing technological openness}

Openness along the technological dimension includes open source technologies and standards like Application Programming Interfaces (APIs). These technologies are becoming a core resource in the city data narrative to quickly and cost effectively implement initiatives. Evidence from primary and observation data reveal that local governments are focusing on technological openness to pursue the following objectives: 1) open source systems to accelerate city data initiatives by replication; 2) open standards for local governments to ensure they have access to their data stored in procured IT systems, 3) open standards for data sharing initiatives across the smart city data ecosystem.

The current state of city data presents scenarios where London local authorities face challenges to access their data that is stored in systems procured from large IT vendors and, at times, having to pay to access data. This has ignited a change in the ecosystem with local authorities coming together to create an open supplier community to prevent vendor lock-in and ensure access to data and interoperability. As informed by an interviewee from a local government:

"there is a common theme of open standard and open APIs to make the digital and data product market more innovative and flexible, else the suppliers will end up putting in energy on something that has no value."

The analysis identified specific examples of open source technologies widely adopted in the ecosystem in the form of open source codes, platforms and licenses. Where open source codes allow for easy replication, standardisation, and validation of data products (e.g. predictive analytics data model), it also enables to upskill local government staff on data science and analytics. However, the benefits of open source tools might come with limitations in transferable knowledge management practices, as an information manager in a local authority explains:

"these days many councils are going down that route of open source as it saves money. We have access to open source, but we don't use it a lot because if we start using it and develop things then we need to have a developer who understands coding, and we don't have this at the moment as they are very expensive. Also let's say we have something in open source and developer leaves, then I have nobody, and if I bring in a new developer, he will first have to learn how previous developer coded it. So, for me it is a risk and I will not take this up to manage it...as a developer you take the knowledge away with you." 
Management and retention issues were mentioned in several aspects of the case study during interviews with most data officers in London local authorities, the GLA or the developer communities involved in smart city projects.

\subsubsection{Managing organisational openness}

All primary, secondary and observational data in this study pointed to the open work practices and principles to orchestrate city data initiatives. Driven by the 'Local Digital Declaration'2 coordinated by the central government in July 2018, a number of local and regional governments have committed to implementing open work practices. Interviews with informants from both local government and GLA highlighted that organisational openness involves being open to ideas and transparent about ongoing projects and procurements, success / failures, particularly in the context of government interactions with vendors and SMEs.

Illustrative initiatives of such work practices are the London report card by the GLA that demonstrates the progress of projects; weekly notes and 'wiki' by local government staff; and creation of a 'pipeline' tool that provides a single place for government technology market to understand local authority needs. These activities help create an open environment for joint procurement of technology or data solutions or as explained by a data lead in one of the local authorities:

"truly working in the open helps to signpost the user through the knowledge: the

business benefit, the use need, the design pattern and the code."

Informants also highlighted that open work practices raises a culture change issue when other local authorities demonstrate a "what's in for us attitude" and stating different priorities to address. Different aspects of this tension were mentioned in several interviews and captured through observation data in conference sessions. There is also a tension in being open in terms of the value exchange between government and SMEs - as highlighted by an SME manager:

"we are trying to sell products and ideas to a shrinking market with shrinking

budgets ..... also struggle to find the right person within government to talk to."

Another aspect of openness was identified by interview participants from all parts of the ecosystem in initiatives like 'city data hackathons' and 'civic innovation challenges'. Such activities were helpful to practice openness both in terms of engagement and discussing the critical elements of delivering value from city data.

Organisational openness is directly connected to the London Datastore managed by the GLA. The open government narrative illustrates organisational openness through publishing open data as a straightforward activity. However, the structural complexity of London is reflected in the complexity of open data and its management practices across the ecosystem. The data that are published in the London Datastore are mainly London-specific datasets that appear in the national data portal and aggregated analytical data (summary statistics) from the GLA. Most London Boroughs have their own individual open data portals and do not directly publish data in the Datastore (see Figure 2). An interview participant explained this tension as one of orchestrating openness of data practices and potential use cases:

${ }^{2}$ https://localdigital.gov.uk/declaration/ 
"The benefit of having our portal is that we put that data in the context of where it is useful because data portal like London Datastore is useful to an analyst. It is not useful to most frontline services, NGOs and commissioners....... if London Datastore wants to go pan-London they need to identify topics and themes on which data can be collected from each borough and be combined and compared, because that is where the value is."

\subsection{Diffusion}

'Openness' allows for accelerating city data initiatives by avoiding duplication of efforts in the ecosystem, while 'diffusion' allows for sustaining initiatives by embedding new practices that develop capabilities in data literacy and facilitate a data culture. Conceptually these practices can be divided into learning and knowledge mobility, and legitimacy and trust building activities.

\subsubsection{Learning and knowledge mobility}

Practices of shared learning were prominent within the smart city data ecosystem. Data literacy was a special focus and not limited to analytical and data engineering skills but also including an understanding of data-policy context amongst managers and leaders. Orchestration of learning practices was an important element in light of the challenges faced by all entities within the city. Most local authorities recognised either lack of advanced data science skills or difficulties in developing them and sustaining teams. This has been a common theme of consideration in industry events and other sources of data where different reasons have been identified as: competition from private sector in recruiting and retaining data experts, austerity and resource constrains, outsourcing data and technical skills not allowing for sustained learning and the rapid evolution of the area making it difficult for leadership to follow developments. With the unavoidable emphasis on developing data literacy skills in-house for local authorities, knowledge sharing, and collaborative practices became a joint point of attention within the ecosystem.

From an orchestration perspective, this resulted in several formal and informal initiatives. Ad hoc structures like the 'Borough data partnership'3 by the GLA or informal data groups within councils with support from senior leadership assisted in spreading awareness of data science skills. Most of these efforts were coordinated across 'insight' and 'intelligence' departments within local authorities and participation was mostly voluntary. Voluntariness promoted genuine learning, but also limited the impact.

Another successful practice during the study was changing the work practices of data science teams to co-locating data scientists and the analytics team with frontline workers. Interview participants agreed that the new teams embedded technical skills with communication management and domain knowledge. For example, a data science team in one of the councils was successful in training their predictive analytics data model only when they engaged and worked together with the frontline staff delivering those services. While this work

${ }^{3}$ https://data.london.gov.uk/borough-partnership/ 
practice is known to improve the matching of technical and domain knowledge, in the case of local authorities it also brought together the institutional knowledge held in various departments of the council, which goes beyond the specific context of working on a service or dataset.

Further, informants highlighted that the efforts to centralise and decentralise analytical capacity across London local government has failed tremendously, and there is a need to implement flexible alternatives to convene data analysts on a data project. As noted by a senior officer from London city administration,

"building the capabilities of people to think beyond their profession and to work in teams is essentially the challenge of 21 century workforces."

This has been further consolidated in interviews with data managers in London local government who suggest that the implementation of analytical networks across the organisation is crucial to enable creativity and ensure getting the right skills as and when needed.

\subsubsection{Legitimacy and trust building}

Empirical data highlights that getting senior leadership buy-in and investment in city data initiatives is risky, primarily because they lack a clear ROI model. As explained by an interviewee of the Smart London Board:

"You cannot isolate enough the variables, so it's difficult to attribute just the data model to save the X amount."

There is a tension in balancing, on one hand the argument that data sharing and data science initiatives will enable delivery of cheaper and efficient city services, and on the other hand, the scepticism around investing time and resources in order to achieve these benefits.

Evidence from most interviews with data officers in London local government and city administration highlighted that government organisations are implementing pilots and an agile approach to deliver city data projects in efforts to win support. This has allowed entities to legitimise the value of the data project to the wider ecosystem, incorporate their feedback and iterate on it. For instance, piloting the LODA (London Office for Data Analytics) allowed to demonstrate the potential of cross-sector data collaboration to the wider city data ecosystem and unravelled the key antecedents needed for its successful implementation.

Further, interviewees revealed that the practice of prioritising projects for 'quick wins' has helped data strategists and innovators, legitimise the value of and build momentum for public sector data science in the wider smart city data ecosystem. A snippet from the interview data with a data strategist in London local government well describes this as:

"There was quite an effort to kind of promote what I call the art of the possible..... the first data scientist started by basically joining existing projects in the council and then saying that how can we do that better with different forms of data or different data science techniques. So, we started doing that and then one by one we started winning over project sponsors, various services and departments and then we went from 2 people in Oct 2016 to now 6 people." 
Informants also suggest guidelines for prioritising projects such as starting with projects that do not require personal data, or prioritising projects on which data of relatively sufficient quality is available. This more focused and boundaried approach helped to gain initial legitimacy for unfamiliar practices.

It is noteworthy, that the initial investment to experiment and demonstrate value, as data suggests, comes from either some stimulation fund from central or regional government or from within local government (the evidence of the latter being limited to very few local government organisations). The challenge of getting the initial investment still remains for major local government bodies in London, more so at a time with massive budget cuts.

\subsection{Shared vision}

Shared vision is a mature element of orchestration wherein a central and visible orchestrator formulates a set of agendas to be addressed based on the requirements across the ecosystem and operationalises it. In the case analysis, 'shared vision' was underpinned by implementing the structures to brainstorm and prioritise agendas, envision opportunities and risks and designing activities that allow for the development and operationalisation of shared vision across the ecosystem.

\subsubsection{Governance tools}

Working towards shared vision in the ecosystem demands structures that enable its effective governance and operation. Having an oversight becomes increasingly important in such collective and integrated ecosystems. Evidence from secondary and primary data sources suggest the set-up of structures like decision boards to ease the process and steer efforts towards the desired outcomes. For instance, the Smart London Board represents expertise from each domain in the city like public sector data or $5 \mathrm{G}$ and is run by the GLA to advise the Mayor. Interviews with council service directors revealed that similar boards are established at certain London local government organisations with representation from local businesses and academia to provide suggestions and oversight to orchestrate the local community.

These collective entities, similar to business networks, are characterised by a set of norms that define the vision and shared ways of working. In the context of London's smart city data ecosystem, this has been structured with the establishment of an institution to strategise citywide collaboration. The institution named LOTI (London Office for Technology and Innovation) is essentially a 'coalition of the willing and the able' London local government organisations to define a set of norms and agenda to shape the smart London city data narrative alongside the GLA. The establishment of this structure has been important because the GLA does not hold much direct authority in how different parts of the ecosystem function.

Although in its early stages at the time of this study, interviews with data officers and open data portal managers across London's councils suggest the scoping of incentives like an open data accelerator to orchestrate publishing more datasets on the London Datastore. Other coupling initiatives aimed to a set rules by scoping an open data charter for London and providing incentives in the form of secondments to boost training in data science for local 
government staff. The latter incentive was prominent in the observation data and was consistently mentioned in industry and local authority events.

The above illustrate that developing shared vision is about implementing governance structures that support engagement and agreement on norms and deciding on deliverables within the ecosystem. This was achieved by considering the structures to allow for engagement, support, incentives and governance of the ecosystem.

\subsubsection{Central co-ordinating structures}

'Shared vision' was also operationalised through the set-up of a central co-ordinating structure for the ecosystem that provided support in core areas of capability development. The biggest and most important milestone in the context of London city data was witnessed with the development of a program as part of the LOTI institution to operationalise city wide data collaboration - the 'City Analytics Program'. Developed from the learnings of the LODA (London Office for Data Analytics) pilot, the program is in charge of leading data collaboration business models of the city through a series of projects involving different actors in the ecosystem - London local government, entrepreneurs, start-ups, universities, research groups, and others. By setting up such a central program that is facilitated by GLA, the program provides support in four core capability areas that are project management, data science, technology and legal. It is interesting to note that the board of the 'City Analytics Program' contains representation only from public sector alongside academia, that is, from the GLA, London local government, and universities.

The analysis revealed a structured approach by the GLA to provide support in data science to the wider data ecosystem through the set-up of the 'Data Academy'. This structure leverages the data science capacity within GLA and is intended to more formally reach all of London's 33 local councils. However, this also raises issues. One interviewee involved in the organisation of this initiative mentioned:

"We are pushing the boundaries of our own understanding. I think some of colleagues will feel uncomfortable standing up and running a course on it because we feel we are not understanding it - one thing is writing code for instance to solve a problem and it's another thing training people on it .... while we are confident, we are doing it in the best way and are very confident about that, explaining that to other people is different."

Moreover, during the course of this study, analysis reveals practices of co-design as an extremely important antecedent to central co-ordinating structures. Largely captured in secondary and observation data sources, the ongoing project of developing a hub for London's planning data presents an example where GLA alongside 33 London local government authorities are codesigning the planning data standards to ensure that their IT systems interoperate. This project is crucial to provide holistic pan-London planning data to inform policy and decision making in the city.

Overall, the above central co-ordinating structures illustrates the collaborative ecosystem approach beyond the competencies of local government to address challenges which are best tackled together. 
*** This is the authors' version - the final version is available via the journal website https://www.sciencedirect.com/science/article/pii/S0040162519312314 ***

\subsection{Presence of individual and collective orchestrators to manage multi-layer} tensions in the smart city data ecosystem

The complex institutional environment in London has resulted in multi-layer tensions that pose challenges in bringing about modest changes and coordinating data initiatives across the ecosystem. The Mayor's office at the GLA although a metropolitan body has limited power over the London Boroughs and functional bodies. Its key responsibilities are setting the budget, drafting strategies and appointing all or most board members of the functional bodies. Transport for London is subject to direct mayoral control as all its board members are appointed by the Mayor, however the same does not apply to both fire and police services. Therefore, tensions at the Mayor's office start from limits in its power to maintain an oversight role and manage the ecosystem to scale city data initiatives. It is due to these limitations in the institutional structures that the city-wide London Office for Technology and Innovation (LOTI) was set up to operate in collaboration with the London Boroughs with support from the Mayor's office at the GLA. The LOTI presents an example of collective orchestrators stimulating the smart city data ecosystem where the governance and oversight function is provided by chief executives both from London Boroughs and the Mayor's office while the management and operations are overseen by the London Boroughs' staff team.

Another tension prevails at the local government level. The trade-off lies in balancing data analysts' efforts either on statutory reporting to GLA and central government, or on more insight driven data projects. Currently, it is the former where most data analysts' efforts are spent. This trade-off widens the gap between local government priorities and the ambitions of the GLA to foster data collaboration in the ecosystem. Further, the technology underpinning data operations in local government have presented challenges like vendor lock-in that results in lack of access, interoperability, and informed procurement decisions on data collaboration projects. An example of successful orchestration in this context is the 'city data hack' initiative by the Future Cities Catapult. It presents an example to catalyse SME and government relations to embed advanced data analytical capabilities in the ecosystem. This initiative also illustrates the flexible nature of Future Cities Catapult as an individual orchestrator. The catapult was responsible for scoping objectives, arranging funding, obtaining government supporters, as well as supporting SMEs to innovate on a government city data modelling toolkit that addresses data interoperability issues, built in-house by catapult.

At the leadership and data personnel level, tensions were manifold. The senior leadership were faced with a trade-off between investing relatively more either in analytical capacity or frontline staff that are responsible for service delivery, with inclination towards the latter because that is a top most priority for local government and where most expenses are. Within a period of austerity for local government, London Boroughs struggled to nurture the vision of advanced data analytics primarily because investing in and retaining data science skills when there is competition from the private sector proves challenging. In efforts to support local government to deliver data enabled services and build advanced analytical capabilities, the GLA developed the initiative of a 'Data academy'. This presents an example of an individual orchestrator with responsibility of program design and delivery by leveraging the in-house expertise of GLA's Intelligence Unit. 
The above tensions highlight the flexibility and leadership required by orchestrators to be successful in navigating institutional structures in orchestrating city data initiatives.

\section{Discussion and implications}

The discussion builds on the above findings to reflect on the importance of orchestration in an integrated perspective on city data and the subsequent theoretical implications of the study. We conclude with practical and policy recommendations from the lessons learnt from London's city data environment.

\subsection{Towards an integrated view of city data}

The analysis of London's city data highlights how technology and data initiatives are primarily about people and change management in the ecosystem. Current frameworks on smart cities consider constructs of technology, data, governance and institutional rules (Brous et al., 2019; Suzuki and Finkelstein, 2019). The orchestration approach allows to integrate the governance and institutional contexts with the narratives of change management and leadership in data initiatives. The orchestration mechanisms support change management and developing city data capabilities in different ways. 'Openness' allows to develop data innovation capabilities in service delivery and designing of data products; 'diffusion' aids in developing a data culture that manifests itself by facilitating data literacy and demonstrating data leadership; lastly, 'shared vision' enables the design and operationalising of strategy around cross-boundary data collaboration and data access in the ecosystem.

Our findings suggest that critical dimensions of city data are about bypassing hierarchies to build a data culture within the smart city data ecosystem. These activities can enable building capabilities like data visualisation and data integration that have been identified by previous studies (Blazquez and Domenech, 2018). Further to investing in urban data platforms, it is important to address the data culture within the organisations and across the ecosystem by investing in training for upskilling staff and developing data leadership capacity. The findings also put emphasis on the role of local government to adopt policy frameworks that facilitate data enabled initiatives like open source technologies and build in-house data capacity (Barns, 2018).

Although studies on data infrastructures include the social element of collaborative data governance and value networks as a key construct (Brous et al., 2019; Suzuki and Finkelstein, 2019) our findings reframe the attention to the managerial perspective. Within London's large and complex data ecosystem, institutional rules and policy frameworks to develop shared understanding remain critical. Specifically, this was reflected in the difficulties and efforts to establish city wide institutional structures that govern cross boundary data sharing in London. Furthermore, the study showed how effective coordination to scale initiatives and sharing good practice to achieve consistency is the primary intention of involved actors. The large Londonwide scale of the case study revealed certain levels of tensions related to data but also a less competitive environment than Meijer's (2018, p. 203) observation that "The actors in the datapolis try to "win" these games to dominate the future of cities". 


\subsection{Theoretical implications}

The study aims to contribute to our theoretical understanding of orchestration as a mechanism of developing new capabilities within data ecosystems. The above discussion draws our attention to the duality of orchestrator in a smart city ecosystem who plays both the roles of strategist (leader) and manager together, in contrast to only being a strategist (Visnjic et al. 2016). Our findings suggest that orchestrators need to be both visionary as strategists but also be able to scope short term priorities that need to be addressed to enable legitimacy building of city data initiatives. Therefore, it might not be sufficient for principal orchestrators (city governments) to only be responsible for long term visions to implement smart city initiatives (Visnjic et al., 2016). Evidence from the case study highlights that the management function in either of those initiatives was not delegated or outsourced but remained within the remit of the orchestrator. It was further important to note the presence of multiple individual and collective orchestrators within London's data ecosystem that differed in their initiatives within an institutionally connected environment but had mutual interests in data leadership and implementation.

The study illustrates how orchestration in smart city data ecosystems is much different from business and innovation ecosystems (Dhanaraj and Parkhe, 2006; Nambisan and Sawhney, 2011). While open system orchestration is about a central orchestrator supporting each member in meeting their respective objectives (Giudici et al., 2018), the intention of the orchestrator in a smart city data ecosystem is to scale up good practice and strive for consistency in city data initiatives within the ecosystem as a whole. Another key difference with business or network orchestration is the shift of emphasis from value appropriation and ecosystem stability (e.g. Nambisan and Sawhney, 2011; Perks et al., 2017) to developing institutional capacity to achieve consistency through openness, diffusion and shared vision. Our findings extend the concept to suggest that orchestration is a mechanism that addresses multi-layer tensions in the ecosystem to scale and achieve consistency of initiatives that meets ecosystem actors' needs and is beneficial for the sustainability of the ecosystem. This orchestration can be particularly applicable to large metropolitan cities and local governance institutions where the central management of city data cannot remain within one central authority.

\subsection{Policy and practical implications}

The findings of this case study offer recommendations and implications for London and other cities and local authorities. Although our study highlights the efforts of the GLA to adopt an outward looking perspective to facilitate collaboration in the city by setting up structures like 'City analytics program' and 'Borough data partnership'; our findings recommend that there is potential for more simplistic efforts that could have a wider impact on how the ecosystem functions. For example, the standardisation of job roles across London local government. Although a minor and basic one, our findings suggest it is crucial to activate the data ecosystem. Currently, the job title and responsibility description for the same role across 33 London local government organisations varies, making it a confusing landscape for ecosystem members and SMEs to identify who to liaise with. Moreover, councils themselves are not aware of the 
capacity available in-house when implementing projects that require join-up of some departments in the council. This current approach reveals a very expensive, inefficient way of working and also highlights the loss of opportunities in collaborating with the ecosystem. Thus, having the GLA take the initiative of standardising job descriptions in the ecosystem would essentially help build analytical networks across the ecosystem as and when needed on data collaboration projects.

The implications of this study for other cities and local authorities might vary depending on their capacities and institutional structure. An overarching insight, especially for cities with those multi-layered and devolved governance structures, is that a central actor undertakes the dual responsibility of the orchestrator as strategist and manager. City governments as orchestrators with only convening and coordination responsibilities may not be not sufficient to scale up and sustain initiatives in their data ecosystems. For instance, although the GLA tried to spread awareness around the scope of data collaboration and advanced data analytics across London by setting up the 'Borough data partnership', the impact was rather ad hoc with the leadership. Embedding a culture around city data and facilitating a more structured approach to data initiatives and data collaboration in the ecosystem, demands the orchestrator to adopt a manager role. This involves training, scoping short term priorities and building legitimacy in the ecosystem. We thus emphasise the demand for an orchestrator to be flexible in nature to materialise on the premise of city governments as intelligent and well-informed users of technology and data.

\section{Conclusion}

The article developed an orchestration approach to smart city data ecosystems defined as an integrated view of data applications by the various city entities that operate within an institutional environment. The empirical case study of London's city data initiatives illustrated how authorities involved in such ecosystems coordinate data initiatives by orchestrating resources and capabilities. Our study highlights that orchestrating smart city data ecosystems requires managing multi-layer tensions that demand the orchestrators to be flexible in nature. The analysis reveals three elements of orchestration - namely openness, diffusion and shared vision - that influence various levels of management, leadership and culture across the ecosystem to develop institutional capacity to coordinate and scale city data initiatives. Our findings point to the following enabling conditions to orchestrate data ecosystems: replicability of data products, openness in work practices, a culture and leadership of data experimentation and embedded work practices and, finally, institutional capacity.

The article aims to extend our theoretical understanding of orchestration by applying the concept to smart city data ecosystems. The analysis highlighted the presence of both individual and collective orchestrators as well as the dual role of an orchestrator as both a strategist and manager. The case consolidates that, following an initial development of data infrastructures and pilot applications, critical dimensions of city data initiatives turn to people and change management. Within complex local governance environments, this becomes an essential step to navigate through layers of bureaucracies and cultures in fragmented and complex data environments than adopting a 'technology first' driven approach. London's data initiatives 
showed how this creates orchestration challenges for managers to build trust and legitimise activities such as the standardisation of job roles related to data across the ecosystem, the development of a data strategy and mapping skillsets available across the ecosystem.

As a single case study, the potential of this analysis for theoretical generalisation might only apply to specific contexts. Other cities and regional authorities will face different challenges and institutional environments within which they will seek to coordinate data initiatives. London's unique context also relates to a large developer community, the existence of big operational and administrative data and the presence of innovation organisations and hubs that can execute technology and innovation management activities in collaboration with local authorities. Other cities might lack these skills and data sources but face less complexity in scale and institutional structures. Limitations to our understanding of London's city data also come from the data collected as part of this study, which are sufficient for a holistic assessment over the period of two years but cannot capture the full complexity and range of activities within such a large environment.

Our study reiterates the need for an integrated view of city data that corresponds to the current level of maturity and ongoing challenges in many cities worldwide. Future research can look into central coordination activities and the development of structures, skills and capabilities that will deliver the potential of city data. In particular, there is much more to learn about leadership and orchestration in smart city data ecosystems where local governments are called to function in new and interesting ways, which to some extent might be outside their traditional skillsets. More research is needed on orchestration and capability assessments to contribute to our new understanding of the management of smart cities and city data.

\section{References}

Appio, F.P., Lima, M., Paroutis, S., 2019. Understanding Smart Cities: Innovation ecosystems, technological advancements, and societal challenges. Technol. Forecast. Soc. Change 142, 1-14.

Barns, S., 2018. Smart cities and urban data platforms: Designing interfaces for smart governance. City, Cult. Soc. 12, 5-12.

Batty, M., Axhausen, K.W., Giannotti, F., Pozdnoukhov, A., Bazzani, A., Wachowicz, M., Ouzounis, G., Portugali, Y., 2012. Smart cities of the future. Eur. Phys. J. Spec. Top. 214, 481-518.

Berrone, P., Ricart, J.E., Carrasco, C., 2016. The Open Kimono: Toward a General Framework For Open Data Initiatives In Cities. Calif. Manage. Rev. 59, 39-70.

Bhattacherjee, A., 2012. Social Science Research: Principles, Methods, and Practices.

Blazquez, D., Domenech, J., 2018. Big Data sources and methods for social and economic analyses. Technol. Forecast. Soc. Change 130, 99-113.

Bresciani, S., Ferraris, A., Del Giudice, M., 2018. The management of organizational ambidexterity through alliances in a new context of analysis: Internet of Things (IoT) smart city projects. Technol. Forecast. Soc. Change 136, 331-338.

British Standards Institution, 2017. Smart cities - Guide to establishing a decision-making framework for sharing data and information services.

British Standards Institution, 2015. City data survey report for BSI in support of understanding data requirements and standards for smart city initiatives.

Brous, P., Janssen, M., Herder, P., 2019. Next Generation Data Infrastructures: Towards an Extendable Model of the Asset Management Data Infrastructure as Complex Adaptive System. Complexity 2019, 1-17. 
Chatfield, A.T., Reddick, C.G., 2017. A longitudinal cross-sector analysis of open data portal service capability: The case of Australian local governments. Gov. Inf. Q. 34, 231-243.

Chourabi, H., Nam, T., Walker, S., Gil-Garcia, J.R., Mellouli, S., Nahon, K., Pardo, T.A., Scholl, H.J., 2012. Understanding Smart Cities: An Integrative Framework, in: Proceedings of the Annual 45th Hawaii International Conference on System Sciences. pp. 2289-2297.

Clegg, S., Josserand, E., Mehra, A., Pitsis, T.S., 2016. The Transformative Power of Network Dynamics: A Research Agenda. Organ. Stud. 37, 277-291.

Cohen, B., Almirall, E., Chesbrough, H., 2016. The City as a Lab: Open InnovatIon Meets the CollaboratIve Economy. Calif. Manage. Rev. 59, 5-13.

Copeland, E., 2015. Big Data in the Big Apple. Cap. City Found.

Cordella, A., Paletti, A., 2019. Government as a platform, orchestration, and public value creation: The Italian case. Gov. Inf. Q. 36, 101409.

Dagnino, G.B., Levanti, G., Mocciaro, A., Destri, L., 2016. Structural Dynamics and Intentional Governance in Strategic Interorganizational Network Evolution: A multilevel Approach. Organ. Stud. 37, 349-373.

Danneels, L., Viaene, S., Van den Bergh, J., 2017. Open data platforms: Discussing alternative knowledge epistemologies. Gov. Inf. Q.

Dawes, S.S., Vidiasova, L., Parkhimovich, O., 2016. Planning and designing open government data programs: An ecosystem approach. Gov. Inf. Q. 33, 15-27.

Dhanaraj, C., Parkhe, A., 2006. ORCHESTRATING INNOVATION NETWORKS CHARLES. Acad. Manag. Rev. 31, 659-669.

EIP-SCC [WWW Document], n.d. URL https://eu-smartcities.eu/ (accessed 6.17.19).

EIP-SCC, BSI, 2016. EIP-SCC Urban Platform Management Framework.

Estermann, B., Fraefel, M., Neuroni, A.C., Vogel, J., 2018. Conceptualizing a National Data Infrastructure for Switzerland. Inf. Polity 23, 43-65.

Ford, D., 2011. IMP and service-dominant logic: Divergence, convergence and development. Ind. Mark. Manag. 40, 231-239.

George, G., Haas, M., Pentland, A., 2014. Big Data and Management. Acad. Manag. J. 57, 321-326.

Gephart, R.P., 2004. Qualitative research and the Academy of Management Journal. Acad. Manag. J. 454-462.

Giffinger, R., Fernier, C., Kramar, H., Kalasek, R., Pichler-Milanović, N., Meijers, E., 2007. Smart cities Ranking of European medium-sized cities. Cent. Reg. Sci. Univ. Technol. 1-26.

Giudici, A., Reinmoeller, P., Ravasi, D., 2018. Open-System Orchestration As A Relational Source of Sensing Capabilities: Evidence from a Venture Association. Acad. Manag. J. 61, 1369-1402.

Greater London Authority, 2018. Smarter London Together.

Greater London Authority, 2016. Data for London.

Hashem, I.A.T., Chang, V., Anuar, N.B., Adewole, K., Yaqoob, I., Gani, A., Ahmed, E., Chiroma, H., 2016. The role of big data in smart city. Int. J. Inf. Manage. 36, 748-758.

Hong, J.F.L., Snell, R.S., 2013. Developing New Capabilities across a Supplier Network through Boundary Crossing: A Case Study of a China-Based MNC Subsidiary and its Local Suppliers. Organ. Stud. 34, 377-406.

Hong, S., Hyoung Kim, S., Kim, Y., Park, J., 2019. Big Data and government: Evidence of the role of Big Data for smart cities. Big Data Soc. 6.

Huxham, C., Vangen, S., 2000. Leadership in the Shaping and Implementation of Collaboration Agendas: How Things Happen in a (Not Quite) Joined-up World. Acad. Manag. J. 43, 11591175 .

Ishida, T., Isbister, K., 2000. Digital cities : technologies, experiences, and future perspectives. 
Springer-Verlag.

Jacobides, M.G., Cennamo, C., Gawer, A., 2018. Towards a theory of ecosystems. Strateg. Manag. J. 39, 2255-2276.

Janssen, M., Estevez, E., 2013. Lean government and platform-based governance-Doing more with less. Gov. Inf. Q. 30, S1-S8.

Jetzek, T., 2016. Managing complexity across multiple dimensions of liquid open data: The case of the Danish Basic Data Program. Gov. Inf. Q. 33, 89-104.

Jetzek, T., Avital, M., Bjorn-Andersen, N., 2014. Data-driven innovation through open government data. J. Theor. Appl. Electron. Commer. Res. 9, 100-120.

Jucevipius, R., Patašienơ, I., Patašius, M., 2014. Digital dimension of smart city: critical analysis. Procedia - Soc. Behav. Sci. 156, 146-150.

Klievink, B., Neuroni, A., Fraefel, M., Zuiderwijk, A., 2017. Digital Strategies in Action - a Comparative Analysis of National Data Infrastructure Development, in: Proceedings of the 18th Annual International Conference on Digital Government Research (Dg.o 2017). pp. 129-138.

Komninos, N., 2011. Intelligent cities: Variable geometries of spatial intelligence. Intell. Build. Int. 3, $172-188$.

Lee, J.H., Hancock, M.G., Hu, M.C., 2014. Towards an effective framework for building smart cities: Lessons from Seoul and San Francisco. Technol. Forecast. Soc. Change 89, 80-99.

Malomo, F., Sena, V., 2017. Data Intelligence for Local Government? Assessing the Benefits and Barriers to Use of Big Data in the Public Sector. Policy and Internet 9, 7-27.

Meijer, A., 2018. Datapolis: A Public Governance Perspective on "Smart Cities." Perspect. Public Manag. Gov. 195-206.

Miles, M.B \& Huberman, A.., 1994. Qualitative data analysis: An expanded sourcebook, Sage Publications.

Mora, L., Deakin, M., Reid, A., 2019. Strategic principles for smart city development: A multiple case study analysis of European best practices. Technol. Forecast. Soc. Change 142, 70-97.

Moshrefzadeh, M., Chaturvedi, K., Hijazi, I., Donaubauer, A., Kolbe, T.H., 2017. Integrating and Managing the Information for Smart Sustainable Districts-The Smart District Data Infrastructure (SDDI) 1-19.

Moutinho, J.L., Heitor, M., 2007. Building human-centered systems in the network society. Technol. Forecast. Soc. Change 74, 100-109.

Nam, T., Pardo, T.A., 2011. Smart city as urban innovation: Focusing on management, policy, and context, in: Proceedings of the 5th International Conference on Theory and Practice of Electronic Governance. pp. 185-194.

Nambisan, S., Sawhney, M., 2011. Orchestration Processes in Network-Centric Innovation: Evidence From the Field. Acad. Manag. Perspect. 25, 40-57.

Nilssen, M., 2019. To the smart city and beyond? Developing a typology of smart urban innovation. Technol. Forecast. Soc. Change 142, 98-104.

Oparaocha, G.O., 2015. SMEs and international entrepreneurship: An institutional network perspective. Int. Bus. Rev. 24, 861-873.

Paquin, R.L., Howard-Grenville, J., 2013. Blind Dates and Arranged Marriages: Longitudinal Processes of Network Orchestration. Organ. Stud. 34, 1623-1653.

Parmentier, G., Mangematin, V., 2014. Orchestrating innovation with user communities in the creative industries. Technol. Forecast. Soc. Change 83, 40-53.

Pereira, G.V., Macadar, M.A., Luciano, E.M., Testa, M.G., 2017. Delivering public value through open government data initiatives in a Smart City context. Inf. Syst. Front. 19, 213-229.

Perks, H., Kowalkowski, C., Witell, L., Gustafsson, A., 2017. Network orchestration for value platform development. Ind. Mark. Manag. 67, 106-121. 
Pikkarainen, M., Ervasti, M., Hurmelinna-Laukkanen, P., Nätti, S., 2017. Orchestration Roles to Facilitate Networked Innovation in a Healthcare Ecosystem. Technol. Innov. Manag. Rev. 7, $30-43$.

Scott, J.C., Glaser, B.G., 2006. The Discovery of Grounded Theory: Strategies for Qualitative Research.

Shaw, D.R., Achuthan, K., Sharma, A., Grainger, A., 2019. Resilience orchestration and resilience facilitation: How government can orchestrate the whole UK ports market with limited resources - the case of UK ports resilience. Gov. Inf. Q. 36, 252-263.

Shin, D.-H., 2009. Ubiquitous city: Urban technologies, urban infrastructure and urban informatics. J. Inf. Sci. 35, 515-526.

Smart Cities | BSI Group [WWW Document], n.d. URL https://www.bsigroup.com/en-GB/smartcities/ (accessed 6.7.19).

Snow, C.C., Håkonsson, D.D., Obel, B., 2016. A Smart City Is A Collaborative Community: Lessons From Smart Aarhus. Calif. Manage. Rev. 59, 92-108.

Suzuki, L.R., Finkelstein, A., 2019. Data as Infrastructure for Smart Cities, Computing and Networks.

Tsujimoto, M., Kajikawa, Y., Tomita, J., Matsumoto, Y., 2018. A review of the ecosystem concept Towards coherent ecosystem design. Technol. Forecast. Soc. Change 136, 49-58.

Van Zoonen, L., 2016. Privacy concerns in smart cities. Gov. Inf. Q. 33, 472-480.

Visnjic, I., Neely, A., Cennamo, C., Visnjic, N., 2016. Governing the City: Unleashing Value from the Business ecosystem. Calif. Manage. Rev. 59, 109-140.

Vogl, T.M., Seidelin, C., Ganesh, B., Bright, J., 2019. Algorithmic Bureaucracy: Managing Competence, Complexity, and Problem Solving in the Age of Artificial Intelligence. Complexity, Probl. Solving Age Artif. Intell.

World Council on City Data [WWW Document], n.d. URL https://www.dataforcities.org/ (accessed 6.7.19).

Yin, R.K., 2009. Case study research: design and methods. Fourth edition, Applied social research methods series.

\section{Appendix A: Interview questions}

1. Introduction and discussion about the interviewee's role within London city data ecosystem:

- City data projects implemented, position, length of previous experience, broader interests within the scope of the project.

2a. Illustrative questions to London Boroughs

- Does the council publish data? If yes, why is the data not published on LDS?

- To what extent does data sharing happen between LBs and LBs and GLA? How do you prioritise projects that require data collaboration?

- How matured is the data architecture of the council? Are these systems procured or built in-house? If procured, what does the decision-making process look like? 
- What solutions suggest supportive collaboration between the technology industry, developers and the government?

- In cases of shared services, how does it work? Who brings in what capacity?

- What do you think is GLA's role in supporting LBs, and vice versa?

- Are there any examples that you can illustrate where the council has transferred or leveraged skills, good practice in data from other councils?

- For years, councils have centralised and decentralised data analyst capacities in the LB, but this hasn't proved fruitful. Why and how can we differently approach it?

- What is the data capacity in the council like? Is there a data scientist - if not, why? if yes, how did you establish a data science team?

- How did you set up a use case and get the buy-in of leadership on a certain data project?

- Do you think your skills are being used to the fullest by the council? (mainly targeted towards data scientists)

- Are there any practices that are being implemented to upskill staff in data literacy? If yes, explain how?

2b. Illustrative questions to Greater London Authority

- Why was the Borough data partnership set up? What were the incentives for LBs to participate when set up in 2014 ?

- What are the key elements of city analytics program? How is it being set up?

- Why do you think LBs are investing in developing their own portal and not publish on LDS? How can GLA address this problem to co-ordinate the process?

- FBs publish data on LDS, although not consistently. What parts of the platform can they control? How does the transfer and publication of data take place?

- What are the priorities for LDS and how is it being resourced?

- How can proven city data initiatives be scaled across the city?

- What is the funding model for city analytics program and what are the plans for sustainable funding?

2c. Illustrative questions to third sector (catapults, ODI, think tanks)

- On pilot projects, which actor had the major influence and why?

- What practices helped in co-ordinate a multi-disciplinary team of data scientists and engineers, SMEs with government? How did you get government to participate/support/adopt the data product?

3. Closing questions

- What were the lessons learnt?

- What is your understanding of a city data infrastructure? (key building blocks?)

- What are the 3 key challenges in working with city data that you would like to address? 